\title{
Fast Tuneable InGaAsP DBR Laser Using Quantum-Confined Stark-Effect-Induced Refractive Index Change
}

\author{
Marianna Pantouvaki, Cyril. C. Renaud, Paul Cannard, Michael J. Robertson, \\ Russell Gwilliam, and Alwyn J. Seeds, Fellow, IEEE
}

\begin{abstract}
We report a monolithically integrated InGaAsP DBR ridge waveguide laser that uses the quantum-confined Stark effect (QCSE) to achieve fast tuning response. The laser incorporates three sections: a forward-biased gain section, a reverse-biased phase section, and a reverse-biased DBR tuning section. The laser behavior is modeled using transmission matrix equations and tuning over $\sim 8 \mathrm{~nm}$ is predicted. Devices were fabricated using postgrowth shallow ion implantation to reduce the loss in the phase and DBR sections by quantum well intermixing. The lasing wavelength was measured while varying the reverse bias of the phase and DBR sections in the range $0 \mathrm{~V}$ to $<-2.5 \mathrm{~V}$. Tuning was noncontinuous over a $\sim 7$-nm-wavelength range, with a side-mode suppression ratio of $\sim 20 \mathrm{~dB}$. Coupled cavity effects due to the fabrication method used introduced discontinuities in tuning. The frequency modulation (FM) response was measured to be uniform within $\pm 2 \mathrm{~dB}$ over the frequency range $10 \mathrm{MHz}$ to $10 \mathrm{GHz}$, indicating that tuning times of $100 \mathrm{ps}$ are possible.
\end{abstract}

Index Terms-Integrated optics, ion implantation, laser tuning, quantum-confined Stark effect (QCSE), quantum well intermixing (QWI), tuneable semiconductor lasers.

\section{INTRODUCTION}

$\mathbf{T}$ UNEABLE lasers attract interest for future wavelength division multiplexed (WDM) optical networks because of the potential to reduce the spares inventory, increase system flexibility, and reduce system cost. Wide tuning ranges and fast tuning are desirable for a variety of applications such as optical packet switching, remote dynamic bandwidth allocation, optical frequency modulation (FM) or rapid link restoration upon network failure [1]-[3], thereby increasing the network flexibility and resource utilization.

Conventional tuneable lasers utilize current injection in multiple sections to induce refractive index changes in the laser

Manuscript received October 31, 2006; revised July 31, 2007. This work was supported by the United Kingdom Engineering and Physical Science Research Council (EPSRC) under Project Photonics Component Research for Integrated Nanotechnology and Communication Environments (PRINCE).

M. Pantouvaki, C. C. Renaud, and A. J. Seeds are with the Department of Electronic and Electrical Engineering, University College London, London WC1E 7JE, U.K. (e-mail: m.pantouvaki@ee.ucl.ac.uk; c.renaud@ee.ucl.ac.uk; a.seeds@ee.ucl.ac.uk).

M. Robertson and P. Cannard are with the Centre for Integrated Photonics, Ipswich IP5 3RE, U.K. (e-mail: michael.robertson@ciphotonics.com; paul.cannard@ciphotonics.com).

R. Gwilliam is with the Advanced Technology Institute, University of Surrey, Guildford GU2 7XH, U.K. (e-mail: r.gwilliam@surrey.ac.uk).

Color versions of one or more of the figures in this paper are available online at http://ieeexplore.ieee.org.

Digital Object Identifier 10.1109/JSTQE.2007.906046 cavity and tune the lasing wavelength. Distributed Bragg reflectors (DBRs) are typically used to provide filtering of the laser cavity modes and select one mode from the spectrum, as they offer larger tuning ranges relative to distributed feedback lasers. Devices incorporating more complex multiple tuning sections (e.g., periodically sampled grating elements) such as the sampled-grating DBR laser (SG-DBR) [4], the superstructure grating DBR laser (SSG-DBR) [5], and the grating-assisted codirectional coupler with sampled reflectors (GCSR) [6], [7] have demonstrated wide tuning ranges $(>40 \mathrm{~nm})$ and high unwanted mode suppression. However, the tuning speed of lasers based on current injection depends on the carrier density changes required to obtain tuning and is limited by the carrier lifetime in the passive (nonamplifying) sections. Wide tuning ranges require large current changes in the passive sections, resulting in increased switching times, particularly when switching occurs due to a change from high to low current [8], [9]. Tuning times of several nanoseconds are typical for such lasers [9]-[12]. Another consequence of the large current changes required for wide wavelength tuning range is heating of the device. Thermal effects cause significant wavelength drifts of microsecond timescales, which can degrade system performance [13], [14].

An alternative way to induce refractive index changes in the laser cavity is by applying a reverse bias to the tuning sections and utilizing field effects such as the Franz-Keldysh (FK) and the quantum-confined Stark effect (QCSE). These effects are based on the absorption edge change in the presence of an electric field, which affects the complex dielectric constant of the semiconductor material at wavelengths close to the bandgap. The FK effect relates to bulk material, while QCSE occurs in structures containing quantum wells. Switching using these effects is not limited by the carrier lifetime, but by the capacitance of the device, making the technique promising for faster switching times. In addition, the lower amount of current in the laser cavity has the potential to reduce thermal effects associated with high current, and the accompanying problems of wavelength drift.

Wavelength tuning based on the FK effect has been demonstrated within $500 \mathrm{ps}$ across $2.5 \mathrm{~nm}$ wavelength range, in a buttjointed structure realized by selective area epitaxy regrowth [15]. Although an improved design utilizing a superstructure grating was proposed to increase the tuning range, the low refractive index changes induced by the FK effect (in bulk material) ultimately limit the tuning range to $\sim 11 \mathrm{~nm}$ [16]. Using the QCSE is a more promising approach due to the sharper excitonic 


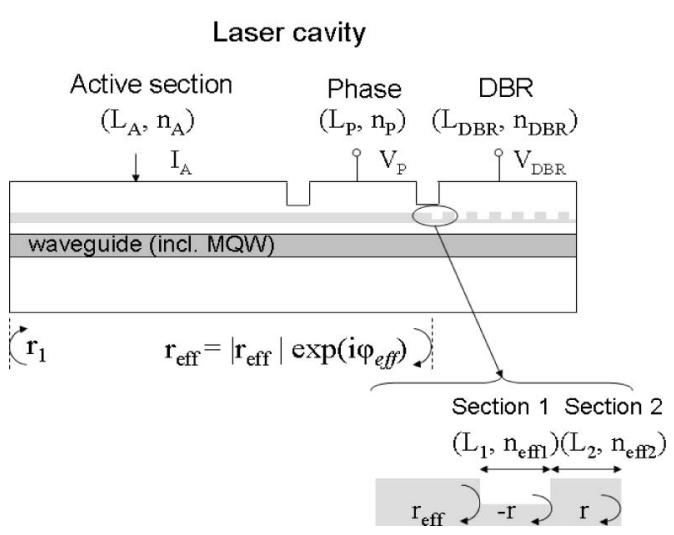

Fig. 1. Schematic of the QCSE-tuned laser.

absorption peak in quantum well structures that results in higher refractive index changes in the material. QCSE tuning was initially demonstrated in an external cavity laser [17], and later, in a tuneable twin-guide DFB laser structure with InGaAsP bulk active region and separate MQW tuning section [18], and in a butt-joined DBR laser with an MQW tuning region realized during separate growth steps [19], resulting in FM responses of $<5 \mathrm{GHz}$. In [20], we demonstrated an integrated two-section GaAs/AlGaAs laser cavity without DBR that was tuned utilizing the QCSE. In this paper, we present a monolithically integrated three-section InGaAsP QCSE-tuned DBR laser structure. Reverse bias is applied to both the DBR and the phase sections to control the lasing wavelength, with constant current on the gain section. The active and passive sections use the same multiple quantum well (MQW) material, while the absorption in the passive sections has been reduced via quantum well intermixing (QWI). QWI based on ion implantation is applied after complete growth of the structure. The technique has the advantage that only one epitaxial regrowth is required after the formation of the DBR, maintaining the simplicity of the fabrication process. Using this device, noncontinuous tuning is demonstrated over $\sim 7 \mathrm{~nm}$ by varying the reverse bias from 0 to $-2.5 \mathrm{~V}$, with a side mode suppression ratio (SMSR) of $\sim 20 \mathrm{~dB}$, and tuning times $<3$ ns limited by the driving source used. FM frequency response measurements indicate that wavelength switching speeds of $100 \mathrm{ps}$ are possible with this device. Significant improvements in the tuning range and SMSR can be accomplished by changing the DBR design.

The remainder of the paper is organized as follows. The QCSE tuning is modeled in Section II using experimental absorption measurements. The device fabrication and quantum well intermixing process are described in Section III. Section IV describes static and dynamic measurements on the QCSE DBR laser. Conclusions from the work are presented in Section V.

\section{QCSE-TUNED LASER THEORY}

The schematic of the QCSE-tuned DBR laser is shown in Fig. 1. The device comprises an active section that provides the gain, and two passive sections, namely the phase and the DBR. The three sections are electrically isolated and individually biased. A constant current is applied to the active section, while the

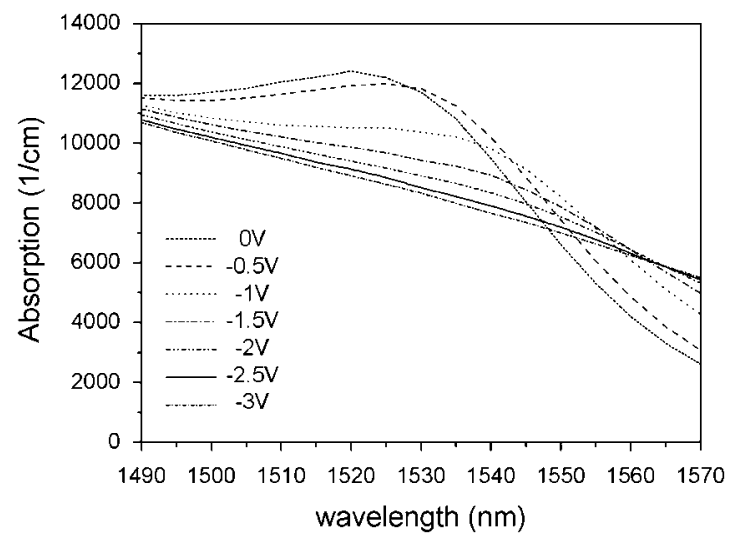

Fig. 2. Absorption spectra for the QW structure of Fig. 6 with electric field.

phase and DBR sections are reverse biased. The waveguide region includes an MQW structure that is common for all sections but is quantum well intermixed in the passive sections to reduce the loss. The grating section is a periodic DBR structure, formed on an InGaAsP layer above the waveguide region. Details on the device structure and fabrication are given in Section III.

\section{A. QCSE Refractive Index Changes}

When a voltage is applied perpendicular to a p-i-n structure with an MQW, the energy bandgap is tilted and the wavefunctions of the electrons and holes in the wells are pulled apart, causing the exciton absorption peaks to red shift and the exciton oscillator strength to reduce. This is the QCSE [21]. The refractive index changes that accompany the absorption changes can be used to modify the light path in a multisection tuneable laser, tuning the wavelength of the output light. Fig. 2 shows the variation of the absorption spectrum of an intermixed, strainbalanced InGaAsP MQW laser structure such as that described in Section III. The absorption spectrum was calculated from photocurrent measurements under the effect of an electric field, with light incident normal to the wafer, using the equation

$$
\alpha_{m}=-\frac{1}{d} \ln \left(1-\frac{h c \rightleftharpoons I_{\mathrm{ph}}}{e \eta P_{\mathrm{ip}}(1-R) \rightleftharpoons \lambda}\right)
$$

where $\alpha_{m}(\lambda)$ is the material absorption, $d$ is the thickness of the quantum wells, $h$ is Planck's constant, $c$ is the speed of light in vacuum, $I_{\mathrm{ph}}$ is the photocurrent, $e$ is the electron charge, $\eta$ is the quantum efficiency, where $\eta=0.9$ was used, $P_{\text {ip }}$ is the incident optical power, $R$ is the reflectivity of the air-semiconductor interface, and $\lambda$ is the wavelength.

The absorption changes observed with increasing magnitude of reverse bias result in refractive index changes that can be calculated by the Kramers-Kronig equation [22]

$$
\Delta n_{m}(\lambda, E)=\frac{\lambda^{2}}{2 \pi^{2}} P \int_{0}^{\infty} \frac{\Delta \alpha_{m}\left(\lambda^{\prime}, E\right)}{\lambda^{2}-\lambda^{\prime 2}} \mathrm{~d} \lambda^{\prime}
$$

where $E$ is the electric field, $P$ is the Cauchy principal value of the integral, and $\Delta \alpha_{m}$ and $\Delta n_{m}$ are the material absorption and refractive index changes, respectively, relative to zero electric field, both depending on the electric field and the wavelength. 


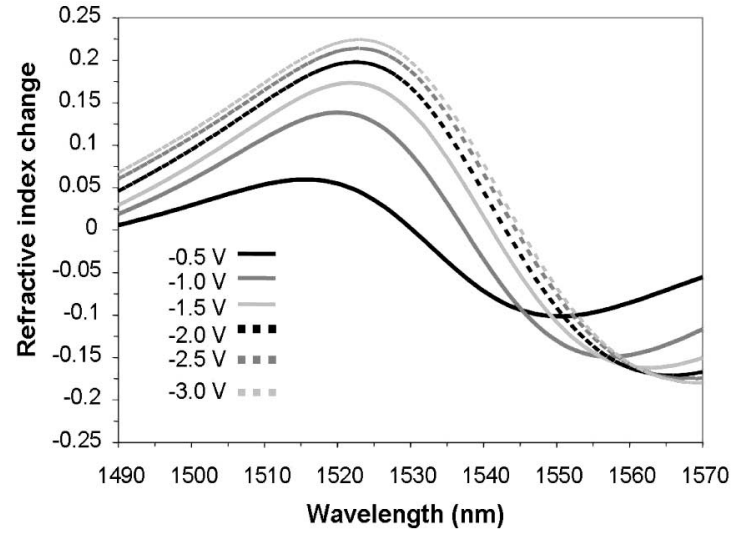

Fig. 3. Refractive index change due to electric field derived from data of Fig. 2. The changes quoted are with reference to the zero-field case.

Fig. 3 presents the refractive index change spectrum $\left(\Delta n_{m}\right)$ calculated from (2) using the absorption spectra of Fig. 2. Though there is a wide spectral range where QCSE-induced refractive index changes could be used, their amplitude varies with wavelength. Significant refractive index changes are observed at longer wavelengths than the exciton absorption peak. For this spectral range, the absorption and absorption changes due to QCSE are lower. A device with intermixed passive sections can take advantage of this as its exciton absorption peak will be blue shifted. In that case, lasing occurs at wavelengths longer than that of the exciton absorption peak of the passive sections. Furthermore, as absorption changes due to QCSE will remain small, tuning of the lasing wavelength is possible without suffering large variations of the laser output power.

\section{B. Tuning Mechanism}

The wavelength tuning due to refractive index changes in the DBR section can be calculated using the transmission matrix theory [23]. The transmission matrix for a single period of the DBR, comprising the two sections of lengths $L_{1}$ and $L_{2}$ as shown in Fig. 1, is

$$
T_{P}=\left[\begin{array}{ll}
T_{P 11} & T_{P 12} \\
T_{P 21} & T_{P 22}
\end{array}\right]
$$

where

$$
\begin{aligned}
& T_{P 11}=\frac{\left(\mathrm{e}^{i \varphi_{1}}-r^{2} \mathrm{e}^{-i \varphi_{2}}\right)}{t^{2}} \\
& T_{P 12}=\frac{r\left(\mathrm{e}^{-i \varphi_{1}}-\mathrm{e}^{i \varphi_{2}}\right)}{t^{2}} \\
& T_{P 21}=\frac{r\left(\mathrm{e}^{i \varphi_{1}}-\mathrm{e}^{-i \varphi_{2}}\right)}{t^{2}} \\
& T_{P 22}=\frac{\left(\mathrm{e}^{-i \varphi_{1}}-r^{2} \mathrm{e}^{i \varphi_{2}}\right)}{t^{2}}
\end{aligned}
$$

and

$$
\begin{aligned}
& \varphi_{1}=\beta_{1} L_{1}+\beta_{2} L_{2} \\
& \varphi_{2}=\beta_{1} L_{1}-\beta_{2} L_{2}
\end{aligned}
$$

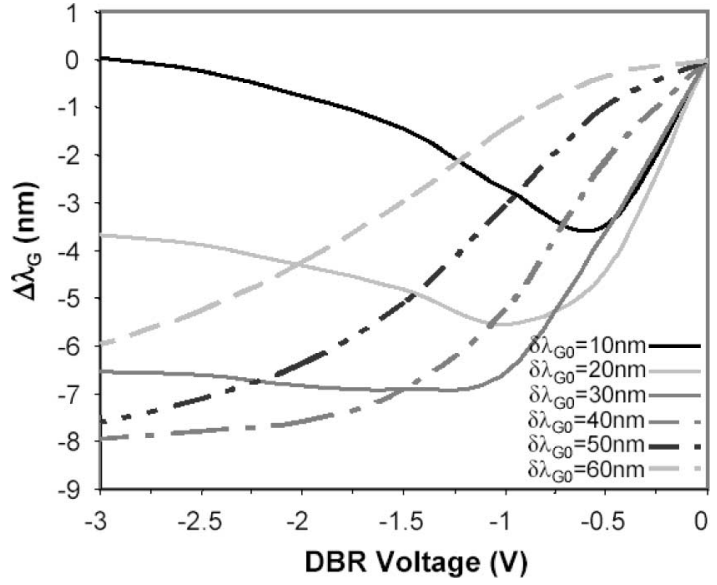

Fig. 4. Tuning of the DBR central wavelength versus DBR voltage for various detunings from the exciton absorption peak.

where $\beta_{1}$ and $\beta_{2}$ are the complex propagation constants in the Sections I and II, and are given by

$$
\beta_{1,2}(\lambda, V)=\frac{2 \pi}{\lambda} n_{1,2}(\lambda, V)-i \frac{\alpha(\lambda, V)}{2}
$$

where $\alpha(\lambda)=\Gamma_{\mathrm{QW}} \alpha_{m}, \Gamma_{\mathrm{QW}}$ is the confinement factor of the QWs, and $n_{1}(\lambda, V)$ and $n_{2}(\lambda, V)$ are the effective indices of the dielectric sections of length $L_{1}$ and $L_{2}$ that constitute the grating. The wavelength and voltage dependence of these effective indices can be written as

$$
n_{1,2}(\lambda, V)=n_{\text {eff } 1,2}(\lambda)+\Delta n_{1,2}(\lambda, V)
$$

where $n_{\text {eff } 1}$ and $n_{\text {eff2 } 2}$ are the effective indices at $0 \mathrm{~V}$ bias of the two segments that comprise the DBR. The wavelength dependence of the refractive indices is calculated as described in [24]. The change of the effective refractive indices with voltage is approximated as $\Delta n_{1}(V)=\Delta n_{2}(V)=\Gamma_{\mathrm{QW}} \Delta n_{m}(V)$, where $\Delta n_{m}$ is calculated from (2) and absorption measurements. In (5)-(8), the reflectivity and transmission coefficients of the interface between the two sections are

$$
r=\frac{n_{2}-n_{1}}{n_{2}+n_{1}} \quad \text { and } \quad t=\sqrt{1-r^{2}} .
$$

The transmission matrix of a DBR of $N$ periods is then given by

$$
T_{G}=\left[\begin{array}{ll}
T_{G 11} & T_{G 12} \\
T_{G 21} & T_{G 22}
\end{array}\right]=T_{P}^{N}
$$

and the reflectivity of the DBR can be calculated from

$$
R_{\mathrm{DBR}}=\frac{T_{G 21}}{T_{G 11}} .
$$

Fig. 4 shows the wavelength tuning $\left(\Delta \lambda_{G}\right)$ of the central wavelength of the DBR $\left(\lambda_{G}\right)$ with applied voltage on the DBR section, calculated as described before. The wavelength tuning is given by $\Delta \lambda_{G}=\lambda_{G}(V)-\lambda_{G 0}$, where $\lambda_{G 0}$ and $\lambda_{G}(V)$ are the wavelength of the DBR at $0 \mathrm{~V}$ and at voltage $V$, respectively. In Fig. $4, \Delta \lambda_{G}$ is calculated for different positions of the DBR central wavelength relatively to the MQW exciton wavelength. This is expressed by $\delta \lambda_{G 0}=\lambda_{G 0}-\lambda_{\text {exc }}$, that is the difference 


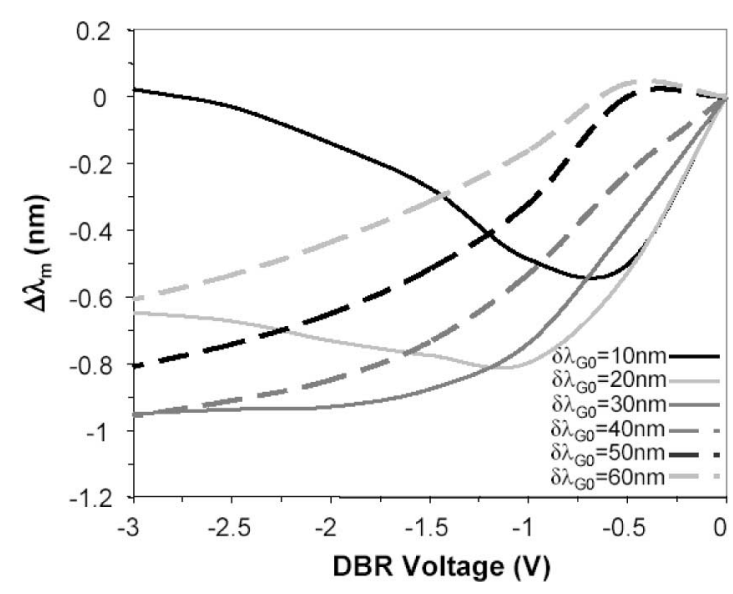

Fig. 5. Tuning versus phase voltage for various detunings from the exciton absorption peak.

between the DBR central wavelength at $0 \mathrm{~V}\left(\lambda_{G 0}\right)$ and the wavelength of the exciton absorption peak $\left(\lambda_{\text {exc }}\right) \cdot \delta \lambda_{G 0}=0 \mathrm{~nm}$ occurs when the DBR wavelength at $0 \mathrm{~V}$ coincides with the wavelength of the excitonic absorption peak. At $\delta \lambda_{G 0}=10 \mathrm{~nm}$, the DBR central wavelength is positioned $10 \mathrm{~nm}$ longer than the exciton peak, etc. The QCSE wavelength dependence suggests that the tuning of the DBR mirror $\left(\Delta \lambda_{G}\right)$ depends on $\delta \lambda_{G 0}$, because of the variations of the refractive index changes with wavelength. However, Fig. 4 shows that $>6 \mathrm{~nm}$ (and up to $\sim 8 \mathrm{~nm}$ ) tuning is possible across $\sim 30 \mathrm{~nm}$ with the structure used in this work, indicating that tuning ranges $>30 \mathrm{~nm}$ could be achieved if the device design was modified to include multiple tuning sections (e.g., SG-DBR).

Having calculated the reflectivity and the phase of the DBR section, the tuning of the lasing wavelength due to the phase section can be found from

$$
\frac{\Delta \lambda_{m}}{\lambda_{m}}=\frac{\left(\Delta n_{A} L_{A}+\Delta n_{P} L_{P}+\Delta n_{\mathrm{DBR}} L_{\mathrm{eff}, \mathrm{DBR}}\right)}{\left(n_{A} L_{A}+n_{P} L_{P}+n_{\mathrm{DBR}} L_{\mathrm{eff}, \mathrm{DBR}}\right)}
$$

where $\lambda_{m}$ is the lasing wavelength of the mode; $n_{A}, n_{P}$, and $n_{\mathrm{DBR}}$ are the effective refractive indices of the active, the phase, and the DBR sections, respectively; $L_{A}$ and $L_{P}$ are the length of the active and the phase sections, and $L_{\text {eff, DBR }}$ is the effective length of the DBR, which is given by

$$
L_{\text {eff, DBR }}=\frac{1}{2} \frac{\mathrm{d} \varphi_{\text {eff }}}{\mathrm{d} \lambda} \frac{\lambda^{2}}{2 \pi n_{G}}
$$

where $\varphi_{\text {eff }}$ is the phase of the DBR reflectivity, as shown in Fig. 1. For constant current applied to the active section and constant voltage on the DBR, this can be written as

$$
\frac{\Delta \lambda_{m}}{\lambda_{m}}=\frac{\Delta n_{P} L_{P}}{\left(n_{A} L_{A}+n_{P} L_{P}+n_{\mathrm{DBR}} L_{\mathrm{eff}, \mathrm{DBR}}\right)} .
$$

Fig. 5 shows the mode tuning due to the phase section calculated

\begin{tabular}{|c|c|}
\hline p-InGaAs (implantation layer) & $100 \mathrm{~nm}$ \\
\hline p- $\ln \mathrm{P}$ (implantation layer) & $500 \mathrm{~nm}$ \\
\hline p. InGaAs contact & $200 \mathrm{~nm}$ \\
\hline$p-\ln P$ & $1400 \mathrm{~nm}$ \\
\hline$p-\operatorname{InGaAsP}\left(\lambda_{2}=1.3 \mu \mathrm{m}\right)$ (grating layer) & $30 \mathrm{~nm}$ \\
\hline$p-\ln P$ & $170 \mathrm{~nm}$ \\
\hline $\operatorname{InGaAsP}\left(\lambda_{8}=1.3 \mu \mathrm{m}\right) \mathrm{SCH}$ & $10 \mathrm{~nm}$ \\
\hline $8 \times \operatorname{lnGaAsP}\left(\lambda_{g}=1.65\right.$ um) wells & $7 \mathrm{~nm}$ \\
\hline$\& 7 \times \operatorname{lnGaAsP}\left(\lambda_{B}=1.3 \mu \mathrm{m}\right)$ barriers & $14 \mathrm{~nm}$ \\
\hline $\operatorname{InGaAsP}\left(\lambda_{R}=1.3 \mu \mathrm{m}\right) \mathrm{SCH}$ & $10 \mathrm{~nm}$ \\
\hline$n \cdot \ln P$ & $1500 \mathrm{~nm}$ \\
\hline Semi-insulating InP substrate & \\
\hline
\end{tabular}
from (18). It was found that changing the phase from 0 to $-3 \mathrm{~V}$ fine tunes the laser wavelength by $<1 \mathrm{~nm}$.

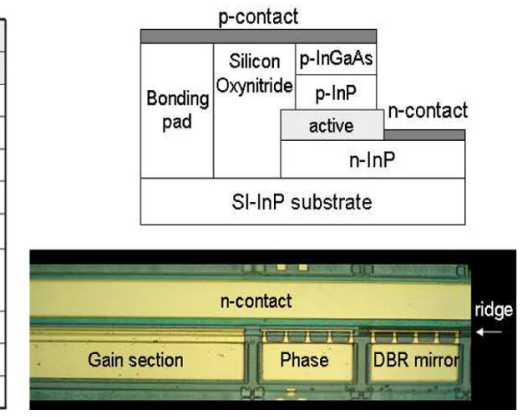

Fig. 6. Wafer structure, schematic cross section, and top picture of the QCSE DBR laser. The top InP and InGaAs implantation layers (in grey) are removed after intermixing and before further processing.

\section{DEVICE DESCRIPTION}

\section{A. Epitaxial Structure}

The structure, the schematic crosssection, and a top picture of the fabricated QCSE DBR laser are shown in Fig. 6. The wafer was grown by metal-organic vapor phase epitaxy (MOVPE) on a semi-insulating InP substrate. The waveguide region incorporates eight 7-nm-wide compressively strained In$\operatorname{GaAsP}\left(\lambda_{g}=1.65 \mu \mathrm{m}\right)$ quantum wells and seven 14-nm-wide tensile strained InGaAsP $\left(\lambda_{g}=1.3 \mu \mathrm{m}\right)$ barriers, sandwiched between two $\operatorname{InGaAsP}\left(\lambda_{g}=1.3 \mu \mathrm{m}\right)$ waveguide layers. Above the waveguide region, a 170-nm-InP spacer layer and a $30 \mathrm{~nm} \mathrm{In-}$ $\operatorname{GaAsP}\left(\lambda_{g}=1.3 \mu \mathrm{m}\right)$ grating layer, both p-doped, were grown. The growth was interrupted at this stage, and a first-order grating was formed on the InGaAsP layer in the area required for the DBR section. The structure was completed by the overgrowth of a $1.4 \mu \mathrm{m}$ p-InP layer, a $0.2 \mu \mathrm{m} \mathrm{p}-I n G a A s$ contact layer, a $0.5 \mu \mathrm{m} \mathrm{InP}$ implantation buffer layer, and a final $0.1 \mu \mathrm{m} \mathrm{In-}$ GaAs implantation buffer layer. Following growth, the tuning sections were quantum well intermixed using ion implantation, as described in the next section.

\section{B. Postgrowth Quantum Well Intermixing}

In the QCSE DBR laser, the gain and the passive sections utilize the same MQW area. As a consequence, the absorption peak of the passive sections is close to the lasing wavelength, introducing significant loss. To reduce the absorption experienced by the light generated in the active section and transmitted through the tuning sections, we used postgrowth QWI to blue shift the bandgap of the passive sections. In QWI, partial mixing of the elements of the wells with those of the barriers can increase the energy bandgap of the wells, and blue shift the photoluminescence peak. The shift depends on the extent of intermixing, which can be varied by changing different parameters of the method used. The advantage of postgrowth QWI compared to selective area epitaxy regrowth is that it is less complex to use, particularly when the passive sections incorporate QWs, while it can also be used to create multiple bandgap detunings on the same wafer in a single QWI step [25].

There are a few techniques that have been used to achieve QWI, such as impurity-free vacancy disordering (IFVD) 
[25]-[28], impurity-induced disordering (IID) [29], laserinduced disordering [30], and ion implantation [31]-[34]. In this paper, we apply shallow ion implantation of $\mathrm{P}^{+}$ions followed by thermal annealing [31], [32]. In contrast to high energy implantation, shallow implantation introduces defects above the cladding, which are then diffused into the quantum wells during annealing. If a sacrificial implantation buffer layer is used, the implantation energy can be chosen so that the ions stop in this layer, which can be removed after annealing, leaving the material free of implanted ions. Compared to deep implantation, shallow implantation can, therefore, maintain better material crystalline quality. In the past, this method was used to intermix partially grown structures, while the remaining p-doped top layers were overgrown after intermixing and removing the implantation buffer layer [32], [34]. This was done to prevent diffusion of the p-dopants into the laser active region during thermal annealing, and hence, performance degradation. In this paper, we use shallow ion implantation followed by rapid thermal annealing at $650{ }^{\circ} \mathrm{C}$ to blue shift the passive sections after completion of growth of the full structure. The method uses implantation buffer layers of different material composition to vary the degree of intermixing, and can be applied to achieve multiple bandgap detunings on the sample during the same QWI step.

When implantation is used for QWI, the bandgap detuning can be varied by changing the implantation energy, the dose, the annealing time, or temperature. In particular, increasing the annealing temperature enhances the intermixing, but also increases the risk for $\mathrm{Zn}$ diffusion. Varying the material of the top buffer layer provides yet another parameter for bandgap detuning control, because the types of defects generated in the material during implantation depend on the material composition itself. Increased bandgap blue shifts have been reported using an InGaAs cap layer, compared to an InP cap, below the $\mathrm{SiO}_{2}$ layer in IFVD [27], [28]. In order to investigate this in the case of shallow implantation and to select the final conditions for the QCSE-tuned lasers, we performed calibration runs using only the InP or both the InGaAs and InP implantation buffer layers of the structure of Fig. 6. To prevent $\mathrm{Zn}$ diffusion and blue shifting in the active section, annealing was performed at $650{ }^{\circ} \mathrm{C}$ temperature.

Samples from the wafer structure described in the previous section were cleaved, and the InGaAs cap layer was removed from half of them, using selective wet etching. The gain section of both types of samples was then covered with $600 \mathrm{~nm}$ $\mathrm{SiO}_{2}$ deposited using plasma-enhanced chemical vapor deposition (PECVD) to prevent intermixing of the area. The samples were subsequently implanted with $\mathrm{P}^{+}$ions at $100 \mathrm{kV}$ energy and $200{ }^{\circ} \mathrm{C}$ temperature, at dose $8 \times 10^{14} \mathrm{~cm}^{-2}$. After implantation, annealing was performed at $650^{\circ} \mathrm{C}$ for various times. During rapid thermal annealing, a clean-polished InP substrate was used as the proximity cap. A comparison of the effect of the top layer composition was also performed. An example of the photoluminescence shift of two passive sections with InP only and InP plus a small InGaAs caps, both annealed for $300 \mathrm{~s}$ at $650{ }^{\circ} \mathrm{C}$, is shown in Fig. 7. The photoluminescence of the active section after the QWI process is also shown for comparison.

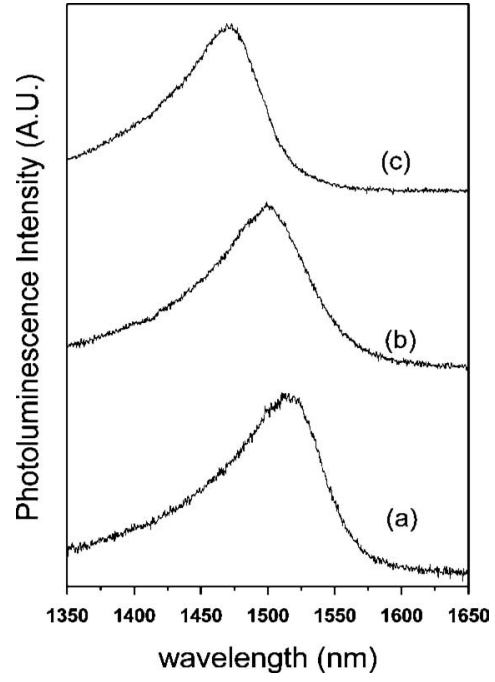

Fig. 7. Room temperature photoluminescence spectra after implantation and annealing at $650^{\circ} \mathrm{C}$ for $5 \mathrm{~min}$. (a) From unimplanted laser section. (b) From passive sections with an InP cap. (c) From passive sections with an InGaAs cap.
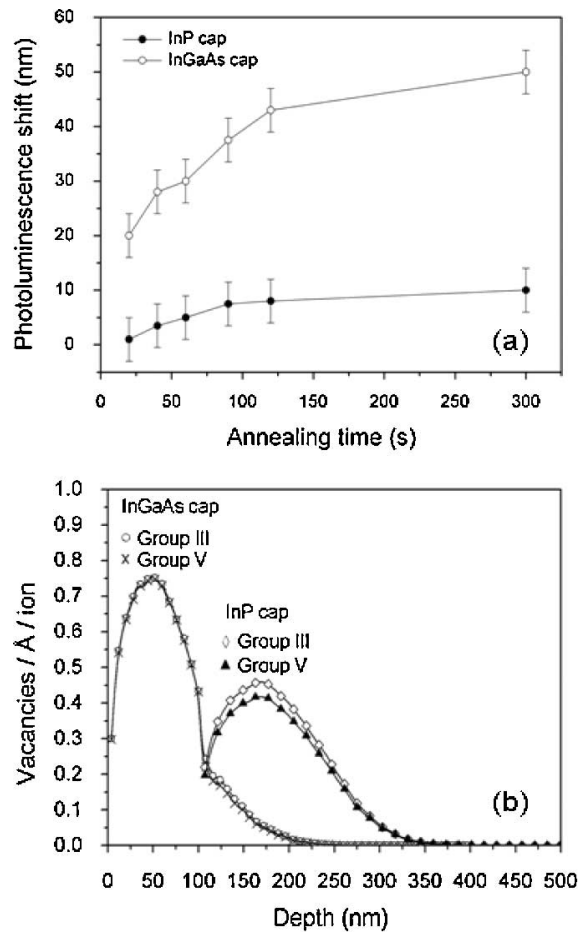

Fig. 8. (a) Wavelength detuning of the photoluminescence peak of the implanted sections as a function of annealing time, for annealing at $650{ }^{\circ} \mathrm{C}$. (b) Simulation of the groups III and V vacancies created during implantation through a top InGaAs and a top InP cap layer.

It was found that, for the same QWI conditions, the samples with the InGaAs cap resulted in higher bandgap detuning of the implanted material, while in both cases, the photoluminescence shift of the unimplanted gain section was negligible. Fig. 8(a) shows in more detail the bandgap detuning for samples with an InP or InGaAs cap as a function of annealing time. There have been a number of theories on how intermixing takes place in InGaAsP, and bandgap detuning has been ascribed to the 

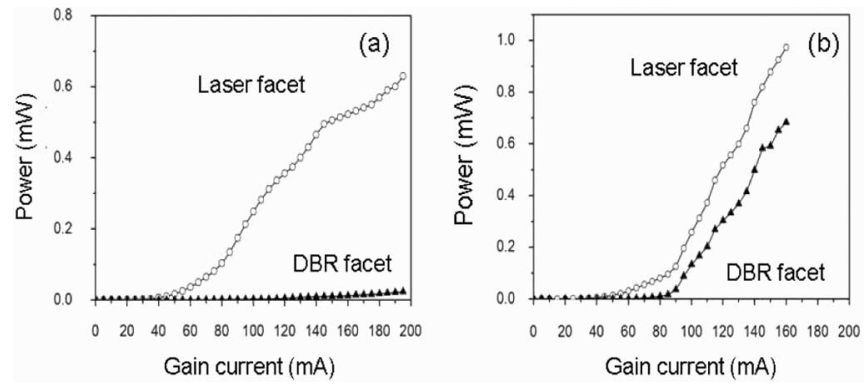

Fig. 9. Laser output power versus gain current plot. (a) For a QCSE DBR laser that has not been intermixed. (b) For a QW intermixed QCSE DBR laser.

interdiffusion of both group III and group V defects [27], [28], [33]. Fig. 8(b) shows the damage induced by implantation with only the InP and with both the InGaAs and InP implantation layers on top of the basic laser structure. The simulation was performed using TRIM [35], which does not account for hightemperature implantation effects, but nevertheless gives an insight of the damage produced during implantation. Although with the InGaAs cap, the peak of the distribution of defects is further away from the active region, the number of calculated defects generated in this case is almost double, as confirmed by the larger detuning measured [Fig. 8(a)].

After the calibration runs for the QWI process, samples with the InGaAs cap that would be processed into QCSE-tuned lasers were covered with PECVD $\mathrm{SiO}_{2}$, implanted using the same conditions as before and annealed at $650{ }^{\circ} \mathrm{C}$ for $90 \mathrm{~s}$. In this way, the photoluminescence peak of the passive sections was blue shifted by $\sim 37 \mathrm{~nm}$ compared to the gain section, in the final devices. Following fabrication, the samples were cleaved to form devices with 1-mm-long gain section, $170-\mu \mathrm{m}$-long phase section, and $160-\mu \mathrm{m}$-long DBR section. The output light from each facet was measured as a function of current in the gain section, for a nonintermixed and an intermixed device, and is shown in Fig. 9. For these measurements, the passive sections were kept at $0 \mathrm{~V}$ bias. Significant reduction of the loss experienced by light output from the DBR facet is observed after QWI. A somewhat higher annealing time could result in even lower loss. The lasing threshold was only slightly increased after QWI, while mode hopping was observed with increasing current in both cases, due to changes in the laser cavity phase with increasing current.

\section{Device Fabrication}

After growth and intermixing, the wafer was processed into ridge waveguide lasers, with three sections, the gain, the phase, and the DBR mirror. Both the $p$ - and $n$-contacts were formed on top of the wafer, while bridges on silicon oxynitride were used to connect the $p$-contact to the bonding pads to reduce the device capacitance. The measured capacitance of the device was $\sim 200 \mathrm{fF} / 100 \mu \mathrm{m}$. A more detailed description of the fabrication procedure can be found in [36].

To use the QCSE as the tuning mechanism, the phase and the DBR sections need to be reverse biased, while the gain section is forward biased. In a monolithically integrated device, where
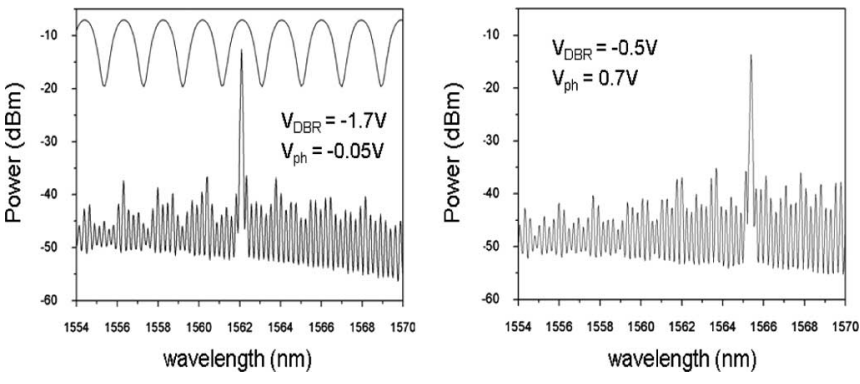

Fig. 10. Examples of the measured output spectra. The dashed line on the first figure represents the spectrum of a $170-\mu$ m-long cavity.

the three sections have a common intrinsic region, good electrical isolation between the sections is necessary. In this work, electrical isolation between the sections was accomplished by removing the top p-InGaAs and $\mathrm{p}$-InP contact layers from a $30-\mu \mathrm{m}$-wide gap between different sections. This resulted in $\sim 15 \mathrm{k} \Omega$ resistance between neighboring $p$-contacts with the gain section unbiased. Higher contact resistance is possible by using ion implantation [15]. Neither of the facets was antireflection (AR) coated for the results presented in this paper.

\section{LASER RESULTS}

\section{A. Static Measurements}

For the tuning measurements of the QCSE DBR laser, the bias current of the gain section was kept at $150 \mathrm{~mA}$, while the reverse bias was varied from 0 to $-2.5 \mathrm{~V}$ on the DBR, and from 0 to $-1 \mathrm{~V}$ on the phase section. Fig. 10 illustrates an example of the spectra measured at the output of the QCSE DBR laser. The variation of the peak output power for all the lasing wavelengths measured was $<6 \mathrm{~dB}$, while the SMSR of the laser was $\sim 20 \mathrm{~dB}$, resulting in nontrivial mode competition. A DBR mirror with stronger filtering characteristic would be desirable. The spectra exhibit modulation of the side modes with period that agrees well with the $170 \mu \mathrm{m}$ length of the phase section (dotted line in Fig. 10). This modulation is attributed to a secondary cavity that is formed between the two isolation gaps, one separating the phase section from the DBR mirror and the second between the phase and the gain sections, resulting in secondary minima in the spectrum, that do not allow for continuous tuning between the laser modes. This is due to the deep etch of the p-doped layers performed in order to electrically isolate the sections, which modified sufficiently the effective refractive index along a distance of $30 \mu \mathrm{m}$, to result in the formation of a secondary cavity.

In Fig. 11(a), the lasing wavelength is plotted versus DBR voltage, while the phase voltage is shown in brackets. The phase voltage was varied to optimize the SMSR. The dotted line in Fig. 11(a) shows an exemplary tuning region of high SMSR while both the grating and phase current were changed. One can note the discontinuities in the tuning map that are mainly due to lateral mode jump and the coupled cavity effects referred to before. In general, the lasing wavelength is moving toward shorter wavelengths across $\sim 7 \mathrm{~nm}$ range, as the DBR voltage 

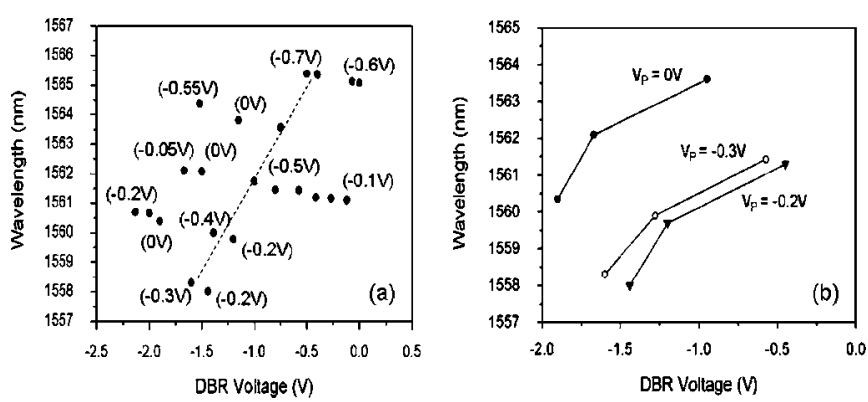

Fig. 11. (a) QCSE DBR laser wavelength versus DBR voltage. The voltage applied on the phase section is shown in brackets. The dotted line shows the general trend with reducing DBR voltage for phase voltage adjusted to maintain high SMSR. (b) Detail of (a), showing the tuning for fixed phase voltage $\left(V_{P}\right)$, for values $V_{P}=0 \mathrm{~V}(\bullet), V_{P}=-0.2 \mathrm{~V}(\boldsymbol{\nabla})$, and $V_{P}=-0.3 \mathrm{~V}(\mathrm{\circ})$.

reduces. The tuning range is comparable to the calculated value of $\sim 8 \mathrm{~nm}$.

The tuning to shorter wavelength, as expected from the calculation shown in Fig. 4, with reducing DBR voltage is also shown in Fig. 11(b), where examples of wavelength tuning for fixed phase bias are plotted. Note that, for this measurement, several mode jumps where observed across the tuning range and the point given are only the one with a good SMSR. As expected from the modeling, the amount of QCSE tuning does not evolve linearly with the applied voltage [Fig. 11(b)]. Again, it agrees well with Fig. 4, for the case that the DBR central wavelength is positioned at $\sim 40 \mathrm{~nm}$ longer wavelength than the exciton peak $\left(\delta \lambda_{G 0}=40 \mathrm{~nm}\right)$, which is the closest approximation to the final device. From Fig. 5, it was expected that the fine tuning induced by the phase section will result in shorter output wavelength when the voltage is reduced. However, from a given voltage, we observed the opposite effect [Fig. 11(b)], that the fine phase tuning was inducing a total shift toward longer wavelength. It was found that this is not due to leakage current. Alternatively, we believe that the main reason for this to occur is that reducing the phase voltage red shifts the absorption peak toward the wavelength of interest, resulting in an increase of absorption in the phase section. This, in turn, reduces the light input to the DBR, which causes a decrease of the photocurrent in the DBR section. Lower photocurrent corresponds to reduced absorption and refractive index changes in the DBR section. This trend competes with the increase in absorption in the DBR due to the decrease of the DBR voltage, resulting in coarse tuning toward shorter and fine tuning toward longer wavelength.

\section{B. Dynamic Measurements}

Dynamic characterization of the QCSE DBR laser was carried out using the configuration of Fig. 12. Two kinds of experiments were performed, the first using a step voltage pulse to measure the switching speed between two lasing wavelengths, while the second was a small signal sinusoidal frequency response measurement. During both experiments, the phase section was kept at fixed reverse bias to simplify the measurement, while the DBR section tuning voltage was applied through a coplanar probe with a $65 \mathrm{GHz}$ bandwidth.

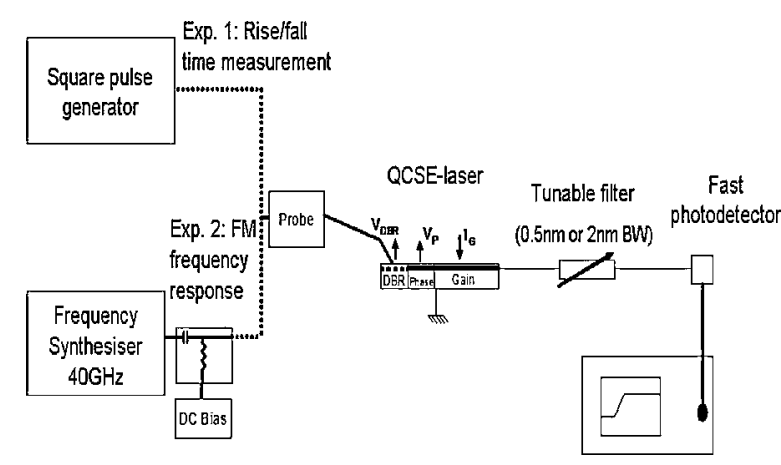

Fig. 12. Experimental configuration for dynamic measurements. The QCSE DBR laser was driven using the square pulse generator for the switching measurements and the sinusoidal frequency generator for the FM response measurement
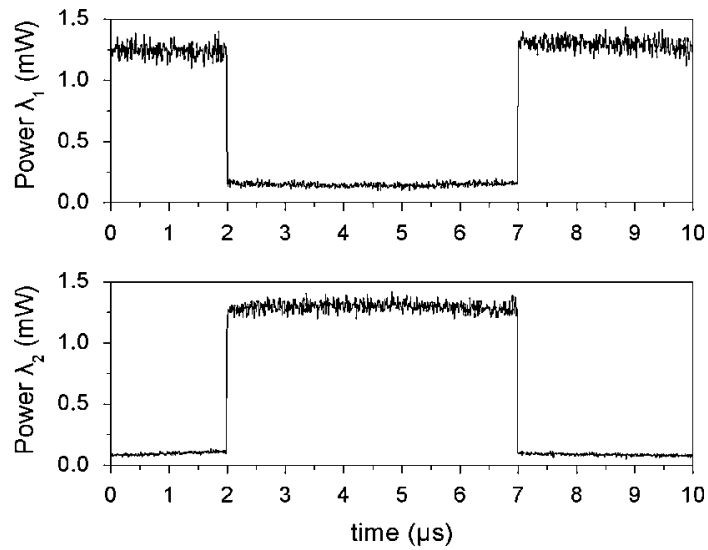

Fig. 13. Oscilloscope traces showing laser output power when switching from wavelength $\lambda_{1}$ and wavelength $\lambda_{2}$.

In the first experiment, the DBR section was driven using a step voltage pulse, between values of the reverse bias that corresponded to two different lasing wavelengths. Since the phase voltage was not varied simultaneously with the DBR reverse bias, only a limited range of switching wavelengths, having good SMSR, were measured. The resulting optical pulse output from the QCSE DBR laser passed through an optical filter of $0.5 \mathrm{~nm}$ bandwidth and the rise and fall times were measured on a sampling oscilloscope with a $60 \mathrm{GHz}$ photodiode. As rise or fall time, we refer to the time required for transition between $10 \%$ and $90 \%$ power at the desired wavelength. Fig. 13 shows the waveforms observed for a switching pulse of $5 \mu$ s duration. For this measurement, the voltage on the phase section was kept at $\sim-0.2 \mathrm{~V}$, and the voltage on the DBR was varied between 0.5 and $-1.2 \mathrm{~V}$, resulting in wavelengths $\lambda_{1}=1561.2$ $\mathrm{nm}$ and $\lambda_{2}=1559.8 \mathrm{~nm}$, respectively. When the optical power at wavelength $\lambda_{1}$ was off, lasing at the second wavelength $\lambda_{2}$ was being switched on. The response is seen to be free of thermal overhang. Details of the leading and falling edges of the driving voltage pulse with corresponding optical power changes are shown in Fig. 14. Although the rise and fall times of the voltage pulse source were approximately $800 \mathrm{ps}$, there was an overshoot of $\sim 0.2 \mathrm{~V}$ of the voltage pulse before it was stabilized at the new state within $\sim 3.5$ ns, as shown in Fig. 14(a). This overshoot 

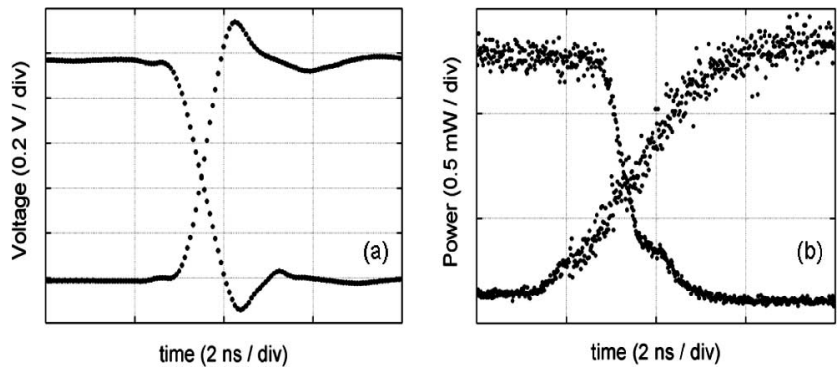

Fig. 14. (a) Input square voltage pulse used for switching measurements. (b) Detail of laser output power at wavelength $\lambda_{2}$.

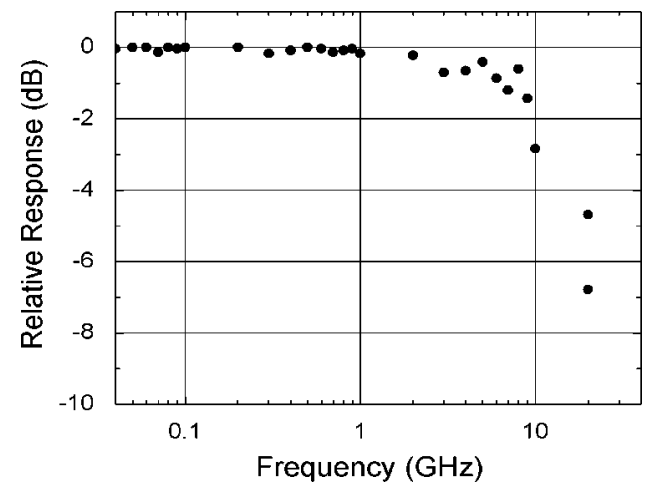

Fig. 15. FM frequency response of the QCSE-tuned DBR laser.

was sufficient to cause the lasing wavelength to switch outside the bandwidth of the filter used in this measurement so that the correct voltage was only achieved after $\sim 3 \mathrm{~ns}$. For the fall time, the voltage merely needs to change sufficiently to tune the laser out of the filter bandwidth, resulting in a much more rapid fall time for the detected optical power. It can be seen that the laser changes wavelength within $1 \mathrm{~ns}$ of the correct voltage being reached.

The second experiment performed for dynamic characterization of the device was a frequency modulation frequency response measurement. In this case, the device was driven by a sinusoidal frequency generator with a peak-to-peak voltage $1 \mathrm{~V}$ at frequencies up to $40 \mathrm{GHz}$. The peak-to-peak value and the dc bias on the DBR section were chosen to keep the laser operation within a continuous tuning region while retaining the largest possible range of wavelength scanned. The optical signal from the QCSE-tuned laser was transmitted through a 2nm-bandwidth optical filter for FM slope detection. During this measurement, the phase section was kept at $0 \mathrm{~V}$ bias. The measured response is shown in Fig. 15. An FM $3 \mathrm{~dB}$ bandwidth of $10 \mathrm{GHz}$ was measured, suggesting that a switching speed of $100 \mathrm{ps}$ is possible with this device.

\section{CONCLUSION}

A monolithically integrated InGaAsP DBR laser using QCSE as the tuning mechanism has been demonstrated. The laser behavior was modeled using transmission matrix equations predicting nonlinear tuning behavior and a quasi-continuous tuning range of $\sim 8 \mathrm{~nm}$ with the absorption peak of the tuning sections blue shifted relatively to the lasing wavelength. Postgrowth QWI based on shallow implantation was used to blueshift the absorption peak wavelength of the tuning sections and reduce the loss. In a nonoptimized device exhibiting coupled cavity effects, noncontinuous tuning across $\sim 7 \mathrm{~nm}$ was demonstrated when the DBR voltage was varied from 0 to $-2.5 \mathrm{~V}$, with $\sim 20 \mathrm{~dB}$ SMSR and $<6 \mathrm{~dB}$ variation of the output power. Switching times $<3$ ns were measured, limited by the switching pulse rise time, while FM frequency response measurements suggest that 100 ps switching times would be possible with such a device.

Several improvements can be made to this device, including using implantation to isolate neighboring sections and suppress coupled cavity effects responsible for tuning discontinuities, improving the mirror design to increase SMSR, aligning the lasing wavelength range with the wavelength range of minimum absorption change in the passive sections to reduce power fluctuations and varying separately the bandgap detuning of the passive sections to reduce tuning due to variations of the photocurrent in the phase section. Finally, considerable refractive index changes were measured across a $\sim 30 \mathrm{~nm}$ spectral range with this device, suggesting that a design utilizing multiple tuning sections could be used to offer wide tuning range.

\section{REFERENCES}

[1] F.-T. An, K. S. Kim, D. Gutierrez, S. Yam, E. Hu, K. Shrikhande, and L. Kazovsky, "SUCCESS: A next-generation hybrid WDM/TDM optical access network architecture," J. Lightw. Technol., vol. 22, no. 11, pp. 2557-2569, Nov. 2004.

[2] C. Bock, J. Prat, and S. D. Walker, "Hybrid WDM/TDM PON using the AWG FSR and featuring centralised light generation and dynamic bandwidth allocation," J. Lightw. Technol., vol. 23, no. 12, pp. 39813988, Dec. 2005

[3] H. Krishnamurthy, K. M. Sivalingam, and M. Mishra, "Restoration mechanisms for handling channel and link failures in optical WDM networks: Tunable laser-based switch architectures and performance analysis," Comput. Commun., vol. 28, pp. 987-999, 2005.

[4] V. Jayaraman, Z.-M. Chuang, and L. A. Coldren, "Theory, design and performance of extended tuning range semiconductor lasers with sampled gratings," IEEE J. Quantum Electron., vol. 29, no. 6, pp. 1824-1834, Jun. 1993.

[5] H. Ishii, H. Tanobe, F. Kano, Y. Tohmori, Y. Kondo, and Y. Yoshikuni, "Quasicontinuous wavelength tuning in super-structure-grating (SSG) DBR lasers," IEEE J. Quantum Electron., vol. 32, no. 3, pp. 433-441, Mar. 1996.

[6] M. Öberg, S. Nilsson, K. Streubel, J. Wallin, L. Bäckbom, and T. Klinga, "74 nm wavelength tuning range of an InGaAsP/InP vertical grating assisted codirectional coupler laser with rear sampled grating reflector,' IEEE Photon. Technol. Lett., vol. 15, no. 5, pp. 735-738, Jul. 1993.

[7] P.-J. Rigole, S. Nilsson, L. Bäckbom, B. Stålnacke, E. Berglind, J.-P. Weber, and B. Stoltz, "Quasi-continuous tuning range from 1560 to 1520 nm in a GCSR laser, with high power and low tuning currents," Electron. Lett., vol. 32, no. 25, pp. 2352-2354, 1996.

[8] E. Buimovich and D. Sadot, "Physical limitation of tuning time and system considerations in implementing fast tuning of GCSR lasers," J. Lightw. Technol., vol. 22, no. 2, pp. 582-588, Feb. 2004.

[9] J. E. Simsarian, A. Bhardwaj, J. Gripp, K. Sherman, Y. Si, C. Webb, L. Zhang, and M. Zirngibl, "Fast switching characteristics of a widely tunable laser transmitter," Photon. Technol. Lett., vol. 15, no. 8, pp. 10381040, 2003.

[10] Y. Su, J. E. Simsarian, and L. Zhang, "Improving the switching performance of a wavelength-tunable laser transmitter using a simple and effective driver circuit," Photon. Technol. Lett., vol. 16, no. 9, pp. 2132-2134, 2004.

[11] C.-K. Chan, K. L. Sherman, and M. Zirngibl, "A fast 100-channel wavelength-tunable transmitter for optical packet switching," Photon Technol. Lett., vol. 13, no. 7, pp. 729-731, 2001. 
[12] R. O'Dowd, S. O'Duill, G. Mulvihill, N. O'Gorman, and Y. Yu, "Frequency plan and wavelength switching limits for widely tunable semiconductor transmitters," IEEE J. Sel. Topics Quantum Electron., vol. 7, no. 2, pp. 259-269, 2001.

[13] B. Puttnam, M. Dueser, and P. Bayvel, "Experimental investigation of the signal degradation in WDM transmission through coherent crosstalk caused by a fast tunable SG-DBR laser," presented at the Opt. Fiber Commun. Conf., Anaheim, CA, Mar. 6-11, 2005, Paper JWA30.

[14] P. Kozodoy, T. A. Strand, Y. A. Akulova, G. Fish, C. Schow, P.-C. Koh, Z. Bian, J. Christofferson, and A. Shakouri, "Thermal effects in monolithically integrated tunable laser transmitters," IEEE Trans. Compon. Packag. Technol., vol. 28, no. 4, pp. 651-657, Dec. 2005.

[15] F. Delorme, S. Slempkes, A. Ramdane, B. Rose, and H. Nakajima, "Subnanosecond tunable distributed Bragg reflector lasers with an electrooptical Bragg section,” IEEE J. Sel. Topics Quantum Electron., vol. 1, no. 2, pp. 396-400, Jun. 1995.

[16] G. Alibert, F. Delorme, S. Grosmaire, S. Slempkes, A. Ougazzaden, and H. Nakajima, "A new tunable laser using a single electroabsorption tuning super structure grating for subnanosecond switching applications," IEEE J. Sel. Topics Quantum Electron., vol. 3, no. 2, pp. 598-606, Apr. 1997.

[17] B. Cai, A. J. Seeds, A. Rivers, and J. S. Roberts, "Multiple quantum well tuned GaAs/AlGaAs laser," Electron. Lett., vol. 25, no. 2, pp. 145-146, 1989.

[18] T. Wolf, K. Drögemüller, B. Borchert, H. Westermeier, E. Veuhoff, and H. Baumeister, "Tunable twin-guide lasers with flat frequency modulation response by quantum confined Stark effect," Appl. Phys. Lett., vol. 60, no. 20 , pp. 2472-2474, 1992.

[19] J. Langanay, E. Gaumont-Goarin, J. Y. Emery, C. Labourie, J. G. Provost, C. Stark, O. Le Gouézigou, and D. Lesterlin, "High FM bandwidth of DBR laser including butt-koined electro-optical wavelength tuning sections," Electron. Lett., vol. 30, no. 4, pp. 311-312, 1994.

[20] X. Huang, A. J. Seeds, and J. S. Roberts, "Reverse bias tuned multiple quantum well ridge guide laser with uniform frequency modulation response," Appl. Phys. Lett., vol. 71, no. 6, pp. 765-766, 1997.

[21] D. A. B. Miller, D. S. Chemla, T. C. Damen, A. C. Gossard, W. Wiegmann, T. H. Wood, and C. A. Burrus, "Band-edge electroabsorption in quantum well structures: The quantum-confined Stark effect," Phys. Rev. Lett., vol. 53, no. 22, pp. 2173-2176, 1984.

[22] D. S. Chemla, D. A. B. Miller, P. W. Smith, A. C. Gossard, and W. Wiegmann, "Room temperature excitonic nonlinear absorption and refraction in GaAs/AlGaAs multiple quantum well structures," J. Quantum Electron., vol. 20 , no. 3, pp. 265-275, 1984

[23] L. A. Coldren and S. W. Corzine, Diode Lasers and Photonics Integrated Circuits (Wiley Series in Microwave and Optics Engineering). New York, NY: Wiley, 1995.

[24] S. Adachi, "Refractive indices of III-V compounds: Key properties of InGaAsP relevant to device design," J. Appl. Phys., vol. 53, no. 8, pp. 5863-5869, 1982.

[25] X. F. Liu, B. C. Qiu, M. L. Ke, A. C. Bryce, and J. H. Marsh, "Control of multiple bandgap shifts in InGaAs-AlInGaAs multiple-quantumwell material using different thicknesses of $\mathrm{PECVD} \mathrm{SiO}_{2}$ protection layers," IEEE Photon. Technol. Lett., vol. 12, no. 9, pp. 1141-1143, Sep. 2000.

[26] D. G. Deppe, L. J. Guido, N. Holonyak, Jr., K. C. Hsieh, R. D Burnham, R. L. Thornton, and T. L. Paoli, "Stripe-geometry quantum well heterostructure $\mathrm{Al}_{x} \mathrm{Ga}_{1-x}$ As-GaAs lasers defined by defect diffusion," Appl. Phys. Lett., vol. 49, no. 9, pp. 510-512, 1986.

[27] J. H. Lee, S. K. Si, Y. B. Moon, E. J. Yoon, and S. J. Kim, "Bandgap tuning of $\mathrm{In}_{0.53} \mathrm{Ga}_{0.47} \mathrm{As} / \mathrm{InP}$ multiquantum well structure by impurity free vacancy diffusion using $\mathrm{In}_{0.53} \mathrm{Ga}_{0.47} \mathrm{As}$ cap layer and $\mathrm{SiO}_{2}$ dielectric capping," Electron. Lett., vol. 33, no. 13, pp. 1179-1181, 1997.

[28] D. H. Yeo, K. H. Yoon, and S. J. Kim, "Characteristics of intermixed InGaAs/InGaAsP multi-quantum well structure," Jpn. J. Appl. Phys., vol. 39, pp. 1032-1034, 2000.

[29] N. Holonyak, "Impurity-induced layer disordering of quantum-well heterostructures: Discovery and Prospects," IEEE J. Sel. Topics Quantum Electron., vol. 4, no. 4, pp. 584-594, 1998.

[30] A. McKee, C. J. McLean, G. Lullo, A. C. Bryce, R. M. De La Rue, J. M. Marsh, and C. C. Button, "Monolithic integration in InGaAs-InGaAsP multiple-quantum-well structures using laser intermixing," J. Quantum Electron., vol. 33, no. 1, pp. 45-55, 1997.

[31] B. Tell, J. Shah, P. M. Thomas, K. F. Brown-Goebeler, A. DiGiovanni, B. I. Miller, and U. Koren, "Phosphorus ion implantation induced intermixing of InGaAs-InP quantum well structures," Appl. Phys. Lett., vol. 54 , no. 16 , pp. $1570-1572,1989$.
[32] M. Paquette, J. Beauvais, J. Beerens, P. J. Poole, S. Charbonneau, C. J. Miner, and C. Blaauw, "Blue shifting of InGaAsP/InP laser diodes by low-energy ion implantation," Appl. Phys. Lett., vol. 71, no. 26, pp. 37493751,1997

[33] J. E. Haysom, P. G. Piva, P. J. Poole, G. C. Aers, S. Raymond, H. Chen, R. M. Feenstra, S. Charbonneau, and I. V. Mitchell, in Proc. Int. Semicond. Insulating Mater. Conf., 2000, pp. 197-204.

[34] E. J. Skogen, J. S. Barton, S. P. Denbaars, and L. A. Coldren, "A quantumwell-intermixing process for wavelength-agile photonics integrated circuits," IEEE J. Sel. Topics Quantum Electron., vol. 8, no. 4, pp. 863-869, 2002 .

[35] J. F. Ziegler, J. P. Biersack, and U. Littmark, The Stopping and Ion Range of Ions In Matter. New York: Pergamon, 1985.

[36] M. Pantouvaki, C. P. Liu, C. C. Renaud, S. Cole, M. Robertson, R. Gwilliam, and A. J. Seeds, "Monolithically integrated QCSE-tuned InGaAsP MQW ridge waveguide DBR laser," presented at the 18th Int. Conf. Indium Phosphide Related Mater. (IPRM 2006), Princeton, NJ, May, Paper MA2.6.

Marianna Pantouvaki received the degree in physics from the University of Athens, Athens, Greece, in 1998, the M.Sc. degree in telecommunications and the Ph.D. degree in electronic engineering from the University College London, London, U.K., in 1999 and 2004, respectively.

She is currently with the Ultra-Fast Photonics Group, University College London, where she is engaged in semiconductor laser amplifiers integrated with saturable absorbers, quantum-confined Stark effect (QCSE) tunable lasers, and optical comb generators.

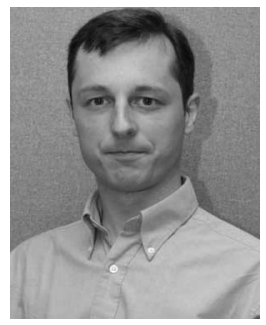

Cyril C. Renaud was born in Paris, France, in 1973. He received the degree of engineering from the Ecole Supérieure d'Optique, Orsay, France, the Diplôme d'Etudes Approfondies (D.E.A.) in optics and photonics from the University Paris XI, Orsay, France, in 1996, and the Ph.D. degree from the Optoelectronics Research Centre, University of Southampton, Southampton, U.K., in 2001.

He was a Project Engineer with Sfim-ODS for a year, where he was enagged in the development of microchips lasers. He then worked with the Optoelectronics Research Centre, University of Southampton, Southampton, U.K., where he was involved in diode-pumped high-power ytterbium-doped fiberlasers, with particular interest on Q-switched system and 980-nm generation. He is currently working on optoelectronic devices and systems in the Ultra-Fast Photonics group, University College London, London, U.K. His current research interests include uncooled WDM sources, agile tuneable laser diode, and monolithic optical frequency comb generator using quantum-confined Stark effect, high frequency photodetectors (UTC, travelling wave), and optical frequency generation systems in the optical and millimeter wave domains (DWDM, THZ).

Paul Cannard received the Graduate degree in 1985 in metallurgy and materials science and the Ph.D. degree in aluminum nitride and its polytypes by atomic imaging TEM from the University College, Cardiff, U.K.

In 1988, he joined the Analytical Group, British Telecom Research Labs, where he used secondary ion mass spectroscopy (SIMS), Auger spectroscopy, transmission electron microscopy (TEM), and scanning electron microscopy (SEM) to study semiconductor and silica-fiber-based technologies. From 1996, he ran a VG80 MBE reactor growing electroabsorption modulator material and studying the potential use of antimonides as mirrors for InP-based VCSELs. In 2000, he joined the Corning Research Centre, and subsequently, CIP in 2004, as Analytical Scientist for epitaxial materials. He is currently with an Aixtron multiwafer reactor to produce planar epitaxial material for a variety of device programmes, and with a Thomas Swan atmospheric reactor for overgrowths of buried heterostructure optoelectronic devices. He holds a U.S. patent, and is a coauthor of more than 20 publications in journals and international conference proceedings. 
Michael J. Robertson received the Ph.D. degree in cadmium sulphide solar cells from Durham University, Durham, U.K., in 1980.

He was with BT Labs, where he worked on GaAs laser reliability. $\mathrm{He}$ then joined BT\&D (now part of Agilent), where led a team that developed a high-reliability planar p-i-n photodiode and successfully transferred this to manufacturing. He was also a part of the team at BT that won the Queen's Award for Technology in 1993 for its work on optoelectronic materials and devices. Since then, he has managed research on expanded mode lasers, semiconductor optical amplifiers, electroabsorption modulators, and optical switches. He has also been at Corning Research Centre, where he led the Epitaxy and Process Development Teams, and is currently the III-V Technology Manager within the Centre for Integrated Photonics (CIP), Suffolk, U.K. He is the author or coauthor of more than 72 papers, has filed ten patents, and has also written a chapter for a book on waveguide analysis.

Russell Gwilliam received the B.Sc. and Ph.D. degrees in electronic and electrical engineering from the University of Surrey, Guildford, U.K. He is currently a Professor of semiconductor process engineering and the Director of Technology for the EPSRC Surrey Ion Beam Centre, Guildford. He has been engaged in developing advanced processes and equipment for both III$\mathrm{V}$ and silicon devices, and is an active Consultant with several international companies. He is a coinventor of the silicon light emission technology on which he founded Si-Light Technologies Ltd. He is the author or coauthor of more than 250 papers.

Prof. Gwilliam is a regular invited speaker at international conferences. $\mathrm{He}$ is a member of the Institue of Physics (IoP), Institute of Engineering and Technology and of several international conference scientific committees.

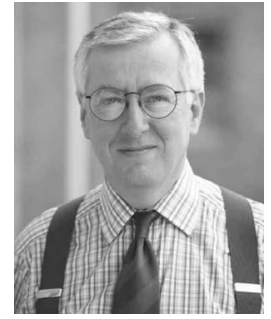

Alwyn J. Seeds (M'81-SM'92-F'97) received the $\mathrm{Ph} . \mathrm{D}$. and D.Sc. degrees from the University of London, London, U.K. From 1980 to 1983, he was a Staff Member at Lincoln Laboratory, Massachusetts Institute of Technology, Cambridge, where he worked on GaAs monolithic millimeter-wave integrated circuits for use in phased-array radar. In 1983, he joined Queen Mary College, University of London, as a Lecturer in telecommunications, and in 1986, moved to the University College London, London, where he is now a Professor of optoelectronics and the Head of the Department of Electronic and Electrical Engineering. He is the author or coauthor of more than 250 papers on microwave and optoelectronic devices and their systems applications, and is also the presenter of the video Microwave Opto-Electronics in the IEEE Emerging Technologies series. His current research interests include microwave bandwidth tunable semiconductor lasers, semiconductor optical modulators, mode-locked lasers, optical phaselock loops, optical frequency synthesis, broadband wireless over fiber access systems, dense wavelength division multiplexed (WDM) networks, terahertz (THz) photonics, and nonlinear optical devices. He is a cofounder of ZinWave, Inc., a manufacturer of wireless over fiber systems.

Prof. Seeds is the Chairman of the Photonics Professional Network of the Institution of Engineering and Technology (U.K.), a Fellow of the Royal Academy of Engineering, and a Member of the Technical Committee on Microwave Photonics of the IEEE Laser and Electro-Optics Society. He has served on the programme committees for many international conferences. 